\title{
Model Predictive Control for Cooperative Control of Space Robots
}

\author{
Somasundar Kannan ${ }^{1, a)}$, Seyed Amin Sajadi Alamdari ${ }^{1}$, Jan Dentler ${ }^{1}$, Miguel A. \\ Olivares-Mendez ${ }^{1}$ and Holger $\operatorname{Voos}^{1}$
}

${ }^{1}$ SnT-University of Luxembourg, 6 rue Richard Coudenhove-Kalergi, Kirchberg, L-1359 Luxembourg

${ }^{a)}$ Corresponding author: somasundar.kannan@uni.lu

\begin{abstract}
The problem of Orbital Manipulation of Passive body is discussed here. Two scenarios including passive object rigidly attached to robotic servicers and passive body attached to servicers through manipulators are discussed. The Model Predictive Control (MPC) technique is briefly presented and successfully tested through simulations on two cases of position control of passive body in the orbit.
\end{abstract}

\section{INTRODUCTION}

The manipulation of passive objects in space has become a key requirement of space missions involving activities such as On-Orbit Assembly, Active Debris Removal missions, Orbital transfer using Space Tug etc. Different methods exists for manipulation of objects in space [1]. The first solution consist of a method where robotic servicer is rigidly attached to the passive body and thrusters of the servicer are directly used to control the motion of the passive object. In the second case the servicer is attached to the passive body through a manipulator and the motion of the passive body is controlled through the manipulator and thruster of the servicer. In the first case the motion of the passive body is directly the motion of the servicer while in the second case the motion of the passive body involves the complex behaviour of the manipulator dynamics and the interaction between the servicer, manipulator and passive body.

In [2] and [3] the problem of capture of an orbital satellite using a space manipulator was studied. In [4] the servicer using multiple manipulators to manipulate a passive body is discussed. In [5] the problem of space assembly was discussed. In [6] a two dimensional problem was discussed where multiple servicers were used to manipulate a passive object. In [1], [7] and [8] the control of servicers with manipulators is discussed where it is assumed that the servicers have already captured the passive object. In [1] a simplified one-dimensional problem was studied where two servicers firmly grasped a passive body. In [1], [7] and [8] the servicers firmly grasped a passive object and a combination of ON-OFF thrusters and manipulators is used to remove the problem of limit cycles and improve the control accuracy with fuel efficiency.

In the current research the problem of orbital manipulation of a passive body using two servicers is discussed. Here only the post-capture phase is studied and for simplicity a one-dimensional problem is solved. The problem of position control of passive body is handled using Model Predictive Control (MPC). The remainder of the article is as follows: First the orbital robotics model is discussed which includes the passive model with rigidly attached thrusters and secondly the combined model of the passive body and two robotic servicers. The applied Mode Predictive Control is briefly discussed followed by the application of the control and modelling techniques to certain experimental scenario through simulation studies. 


\section{ORBITAL ROBOTICS: MODEL}

The modelling aspect of the orbital robotic system is briefly discussed here. The Dynamics of a set of $m$ number of Orbital systems consisting of Robotic Servicer and passive body can be given by [1]

$$
H \ddot{q}+C(q, \dot{q})=\mathrm{Q}
$$

where $q$ is the $n \times 1$ vector of generalized coordinates, that includes $3(m+1)$ positions and $3(m+1)$ Euler angles of the $m$ robot bases, the passive body and also the joint variables of each robot manipulator. Here Q is the $k \times 1$ vector of generalized forces, which includes thrusters, torques from reaction wheels of $m$ robots and joint torques of robot manipulators. $H$ is a $n \times n$ mass matrix and $C$ is a $n \times 1$ vector that contains the velocity terms. In order to further study the problem of orbital robotics we will simplify the system into a one-dimensional problem. We will further study the modelling and control of a passive body directly controlled by thrusters and then analyse the problem of manipulation of a passive object with two orbital robotic servicers.

\section{Passive Body directly controlled by thrusters}

In the current section we will briefly study the simplified one-dimensional model of a passive body directly controlled by thrusters. This is the case when the robotic servicers are rigidly attached to the passive body. Under such condition we can assume that the thrusters of the servicer act directly on the passive body. The simplified one-dimensional model of the passive body directly controlled by thrusters can be seen in Figure-(1). The dynamic equation of a simplified

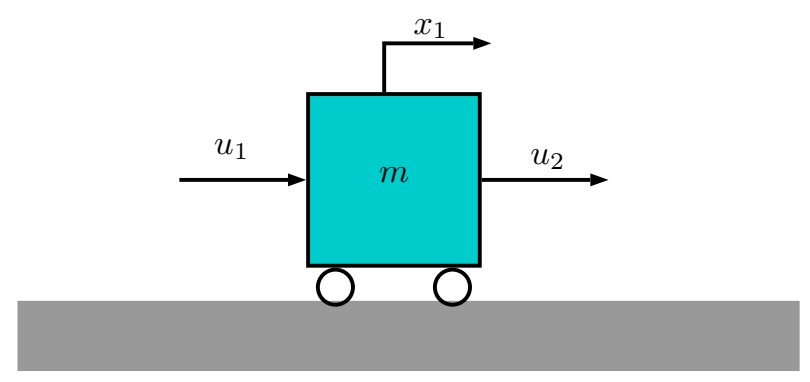

FIGURE 1. Passive body directly controlled by thrusters.

one dimensional model is given by

$$
\begin{aligned}
& \dot{x}_{1}=x_{2} \\
& \dot{x}_{2}=\frac{1}{m}\left(u_{1}+u_{2}\right)
\end{aligned}
$$

Where $m$ is the mass, $x_{1}$ is the position, $x_{2}$ is the velocity, $u_{1}$ and $u_{2}$ are ON-OFF control forces given by

$$
\begin{aligned}
& u_{1}=\left\{\begin{array}{l}
u_{\max }: \text { if } u_{o p t} \geq f_{t} \\
0: \text { if } u_{o p t}<f_{t}
\end{array}\right. \\
& u_{2}=\left\{\begin{array}{l}
-u_{\max }: \text { if } u_{o p t} \leq f_{t} \\
0: \text { if } u_{o p t}>f_{t}
\end{array}\right.
\end{aligned}
$$

where $u_{o p t}$ is an optimal control input generated by the controller, $u_{\text {max }}$ is the force applied by open thruster and $f_{t}$ is deadband on the controller to avoid chattering.

\section{Cooperative Robots and Passive body}

The dynamic model of cooperative orbital robots manipulating a passive body is discussed here. The scenario that is being considered here is that two space robots transport a passive body attached between them through a manipulator 
each from the two space robots. For simplicity only a one-dimensional model is considered as before and further simplifications are made to the manipulator model. The two manipulators are firmly attached between the passive body and the robots and have certain degree of flexibility. This is the case when the links are flexible or links are rigid with flexible joints. The flexible behaviour can be approximated by spring and damper model as can be seen in Figure-2. The dynamic equation of a simplified one dimensional model can be given by:

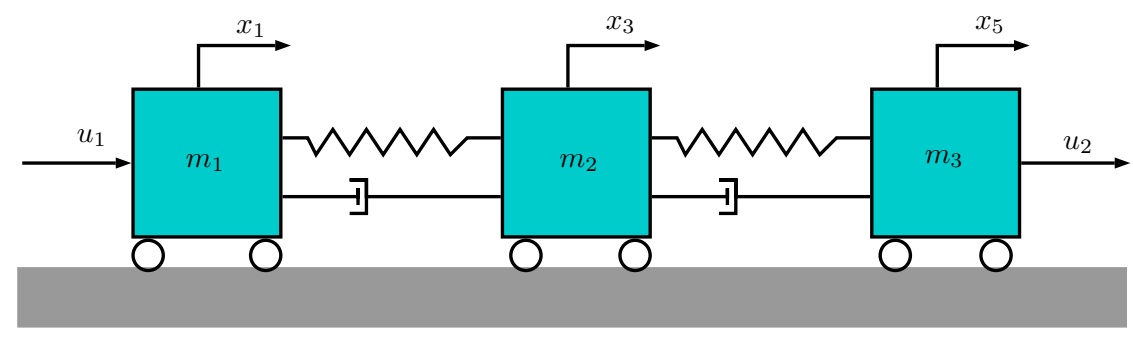

FIGURE 2. Cooperative control of passive body.

$$
\begin{aligned}
& \dot{x}_{1}=x_{2} \\
& \dot{x}_{2}=\frac{1}{m_{1}}\left(u_{1}+k_{2}\left(x_{3}-x_{1}\right)+c_{2}\left(x_{4}-x_{2}\right)\right) \\
& \dot{x}_{3}=x_{4} \\
& \dot{x}_{4}=\frac{1}{m_{2}}\left(-k_{2}\left(x_{3}-x_{1}\right)-c_{2}\left(x_{4}-x_{2}\right)+k_{3}\left(x_{5}-x_{3}\right)+c_{3}\left(x_{6}-x_{4}\right)\right) \\
& \dot{x}_{5}=x_{6} \\
& \dot{x}_{6}=\frac{1}{m_{3}}\left(u_{2}-k_{3}\left(x_{5}-x_{3}\right)-c_{3}\left(x_{6}-x_{4}\right)\right)
\end{aligned}
$$

Where $m_{1}, m_{2}, m_{3}$ are the masses of three bodies, $x_{1}, x_{3}, x_{5}$ is the position vector, $x_{2}, x_{4}, x_{6}$ is the velocity vector, $u_{1}$ and $u_{2}$ are ON-OFF control forces as described earlier. Here $\left\{k_{1}, k_{2}\right\}$ and $\left\{c_{1}, c_{2}\right\}$ are spring and damping constants of the manipulators.

\section{MODEL PREDICTIVE CONTROL}

Here we will briefly discuss the Model Predictive Control (MPC) algorithm used to control the system. The dynamics of the system can be described by [9]

$$
\dot{\vec{x}}(t)=\vec{f}(\vec{x}(t), \vec{u}(t), \vec{p}(t)),
$$

where $\vec{x}(t) \in \mathbb{R}^{n}$ is the state vector, $\vec{u}(t) \in \mathbb{R}^{m_{u}}$ is the input vector and $\vec{p}(t) \in \mathbb{R}^{m_{p}}$ is the vector of time dependent parameters.

The MPC uses an internal model to predict the behaviour of the system over the horizon $\tau=[t, t+T]$ at each time $t$. The control input is determined by minimization of the performance index $J$ subject to constraints $\vec{C} \in \mathbb{R}^{m_{c}}$. To summarize an optimal control problem is solved at each time instant $t$ given by [9]:

$$
\begin{aligned}
& \min _{\mathbf{u}} \quad J \quad=\varphi\left(\vec{x}_{\tau}(T, t), \vec{p}(T, t)\right)+\int_{t}^{t+T} L\left(\vec{x}_{\tau}(\tau, t), \vec{u}_{\tau}(\tau, t), \vec{p}(\tau, t)\right) d \tau \\
& \text { s.t. } \quad \dot{\vec{x}}_{\tau}(\tau, t) \quad=\vec{f}\left(\vec{x}_{\tau}(\tau, t), \vec{u}_{\tau}(\tau, t), \vec{p}(\tau, t)\right), \\
& \vec{x}_{\tau}(0, t) \quad=\vec{x}(t) \\
& \overrightarrow{0} \quad \geq \vec{C}\left(\vec{x}_{\tau}(\tau, t), \vec{u}_{\tau}(\tau, t), \vec{p}(\tau, t)\right) .
\end{aligned}
$$

where $\varphi$ is the terminal cost, $L$ is the running cost and $\vec{x}_{\tau}, \vec{u}_{\tau}$ are the predicted trajectories indexed by $\tau$ in the prediction horizon. In the above optimal control problem the feedback to close the loop is introduced by (15), where the actual state $x(t)$ is used as initial state $\vec{x}_{\tau}(0, t)$. 
In the current MPC implementation the the continuation generalized minimum residual method (CGMRES)[9] is used. The CGMRES method has been successfully tested in [10], [11], [12], [13] and [14] with the help of open source code available from [15]. In the CGMRES method an auxiliary variable method is used to implement the inequality constraint by using slack variables $\alpha$, where [9]

$$
0 \geq C\left(\vec{x}_{\tau}(\tau, t), \vec{u}_{\tau}(\tau, t), \vec{p}(\tau, t)\right)
$$

is converted to

$$
0=C\left(\vec{x}_{\tau}(\tau, t), \vec{u}_{\tau}(\tau, t), \vec{p}(\tau, t)\right)+\alpha^{2}
$$

Finally the solution of the CGMRES method is the optimal control input $\vec{u}_{\tau}$, where only the first control input $\vec{u}(t)=$ $\vec{u}_{\tau}(0, t)$ is applied to the system.

\section{SIMULATION RESULTS}

Here we will briefly discuss the application of Model Predictive Control (MPC) technique to the control of cooperative orbital robots. First we will briefly discuss the position control of a passive object rigidly attached with two satellites and then we will discuss the scenario of a passive body controlled by cooperative robots.

\section{Direct Control of Passive Object in Orbit}

Here we will briefly describe the scenario of direct control of passive orbital object. This is the case where a massive passive object is controlled by two servicer satellites rigidly attached and providing thruster forces. To perform the simulation let us consider a passive mass $m_{1}$ of $90 \mathrm{~kg}$, actuated using $u_{1}$ and $u_{2}$ with $u_{\max }=(0,50)$ and $u_{\text {min }}=(-50,0)$. The scenario is that the passive mass $m_{1}$ has to move from 0 to $25 \mathrm{~m}$ using thrusters. The trigger variable for the actuator is defined by $f_{t}$. The respective running and terminal Cost are given as:

$$
\begin{gathered}
L=\frac{1}{2}\left(\left(x-x_{f}\right)^{T} Q\left(x-x_{f}\right)+u_{1}^{2} r_{1}+u_{2}^{2} r_{2}+u_{3}^{2} r_{3}+u_{4}^{2} r_{4}+g_{1}\left(u_{3}+u_{4}\right)\right) \\
\varphi=\frac{1}{2}\left(x-x_{f}\right)^{T} S_{f}\left(x-x_{f}\right)
\end{gathered}
$$

with the constraint function

$$
C(u)=(u-\bar{u})^{2}-\left(u_{\max }-\bar{u}\right)^{2}+\alpha^{2}, \bar{u}=\frac{u_{\max }+u_{\min }}{2}, \alpha:=f\left(u_{3}, u_{4}\right)
$$

The time varying parameter $p(t)=0$. The other tuning parameters are: $Q=\operatorname{Diag}\left[900,5 \times 10^{3}\right], R=\operatorname{Diag}[1,1,0,0]$, $g_{1}=1, \varphi=0$ with prediction horizon $T_{h}=3$ and sampling rate $T_{s}=0.01$. The simulation results for the current scenario can be seen in Figure-3,4,5,6. The task here is that the Passive body is moved from initial position of $0 m$ to final position of $25 \mathrm{~m}$. In Figure-3 we can see the position control response of the passive body and respectively the velocity can be seen in Figure-4. In Figure-5 we can see the optimal control solution provided by the Model Predictive Control while Figure- 6 shows the ON-OFF actuation response to perform the control objective. The control forces $u_{1}$ and $u_{2}$ are counter acting forces with $u_{1}$ acting as a positive force to push and $u_{2}$ acting as a negative force to stop the displacement of the passive body. The control signals are supplied to the ON-OFF actuator and the response can be seen in Figure-6.

\section{Cooperative Control of Passive Body}

In the current scenario a passive body in the center is displaced by two robotic servicers with thrusters. The schematic of the problem can be seen in Figure-2. For the simulation purpose the mass of passive body $m_{2}$ is $400 \mathrm{~kg}$, which is carried by satellite $m_{1}$ and $m_{3}$ of mass $90 \mathrm{~kg}$ each. The servicers are actuated using $u_{1}$ and $u_{2}$ with $u_{\max }=(0,50)$ and $u_{\text {min }}=(-50,0)$. The target here is that the passive mass $m_{2}$ has to move from 0 to $25 m$ while $m_{1}$ and $m_{2}$ should maintain a distance of $1.5 \mathrm{~m}$ from $m_{2}$. The respective running cost and terminal cost function can be seen as follows:

$$
\begin{aligned}
L=\frac{1}{2}\left(\left(x-x_{f}\right)^{T} Q\left(x-x_{f}\right)+u_{1}^{2} r_{1}+u_{2}^{2} r_{2}+u_{3}^{2} r_{3}+u_{4}^{2} r_{4}\right. \\
\left.\quad+g_{1}\left(u_{3}+u_{4}\right)+w_{g a p}\left(x_{1}-x_{3}-1.5\right)^{2}+w_{g a p}\left(x_{3}-x_{5}-1.5\right)^{2}\right)
\end{aligned}
$$




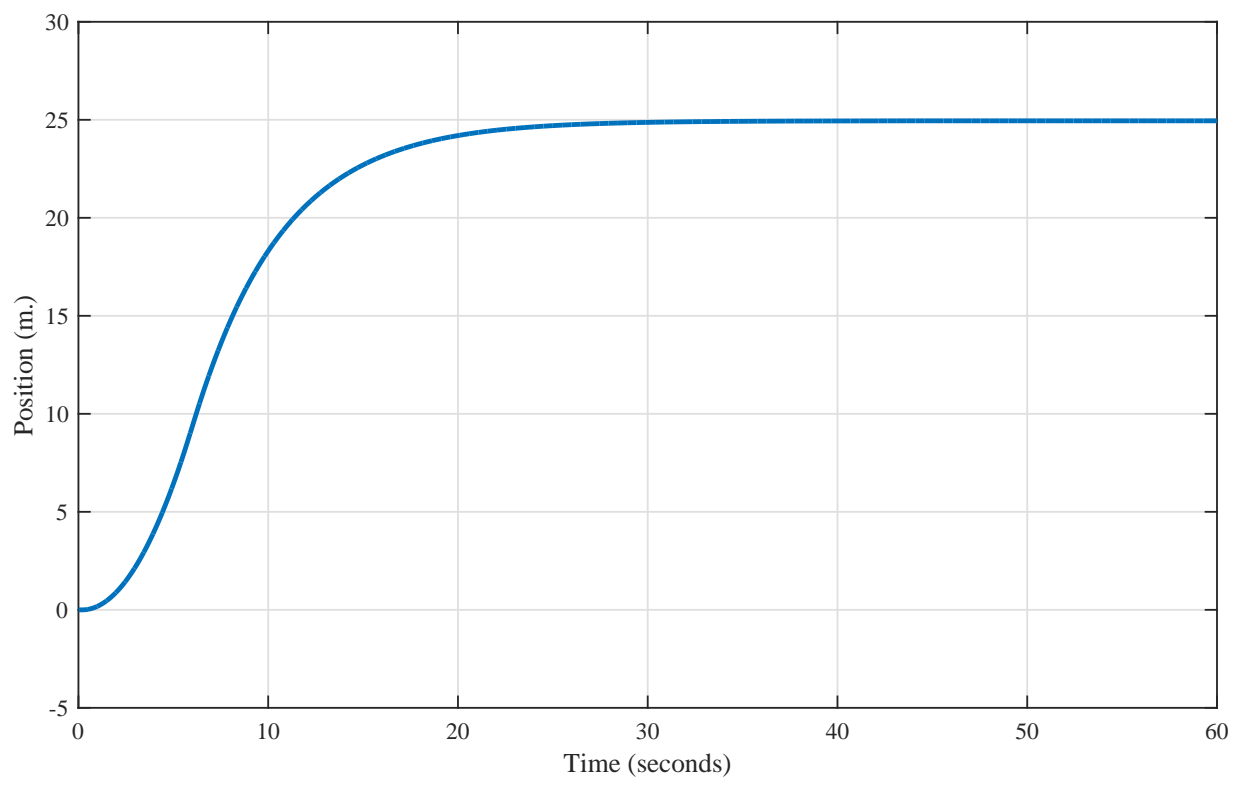

FIGURE 3. Position Control: Passive Body controlled directly by thrusters.

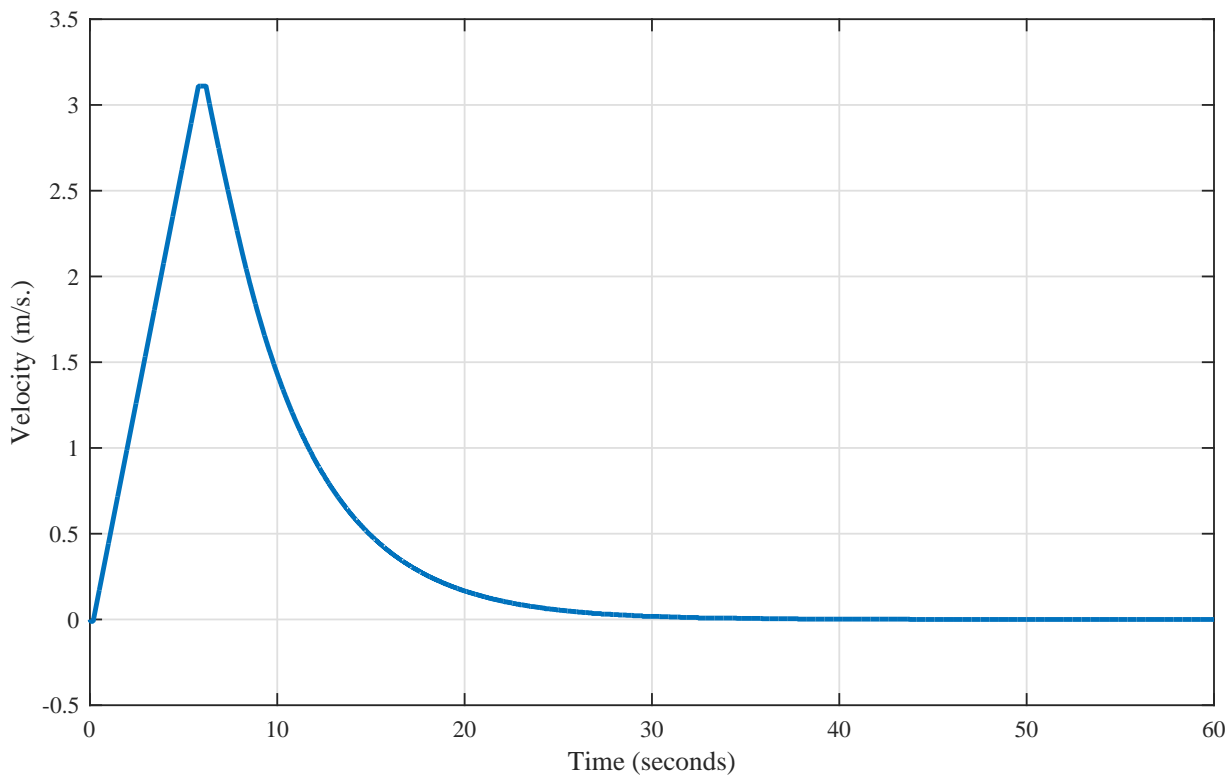

FIGURE 4. Position Control: Velocity of the Passive body.

$$
\varphi=\frac{1}{2}\left(x-x_{f}\right)^{T} S_{f}\left(x-x_{f}\right)
$$

The constraints that are used here can be given by

$$
C(u)=(u-\bar{u})^{2}-\left(u_{\max }-\bar{u}\right)^{2}+\alpha^{2}, \bar{u}=\frac{u_{\max }+u_{\min }}{2}, \alpha:=f\left(u_{3}, u_{4}\right)
$$

and the time varying parameter is $p(t)=0$. The other tuning parameters are: $Q=\operatorname{Diag}\left[4 \times 10^{3}, 1 \times 10^{5}, 4 \times 10^{3}, 1 \times\right.$ $\left.10^{5}, 4 \times 10^{3}, 1 \times 10^{5},\right], R=\operatorname{Diag}[1,1,0,0], g_{1}=10, \varphi=0, w_{\text {gap }}=200, k_{2}=k_{3}=10, c_{2}=c_{3}=1000$ with 


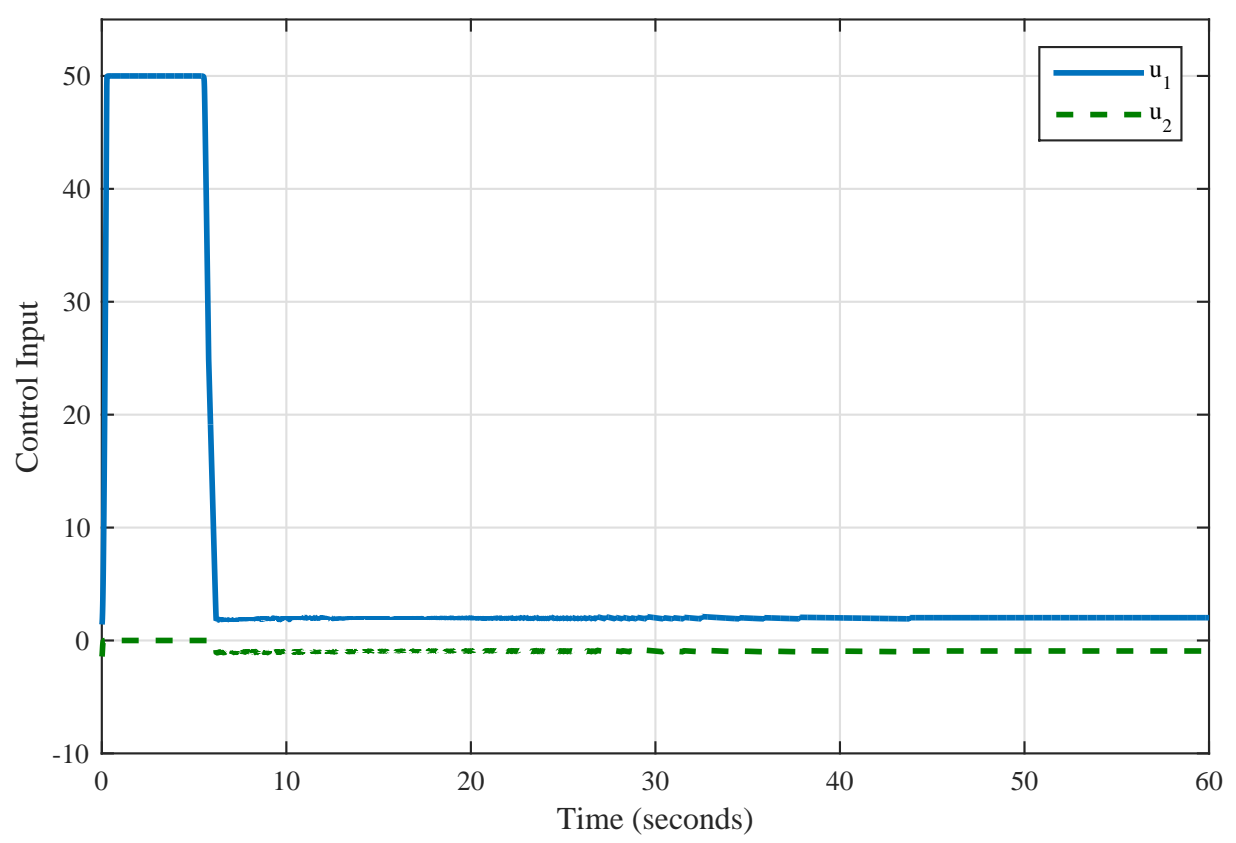

FIGURE 5. Position Control: Control Signal.

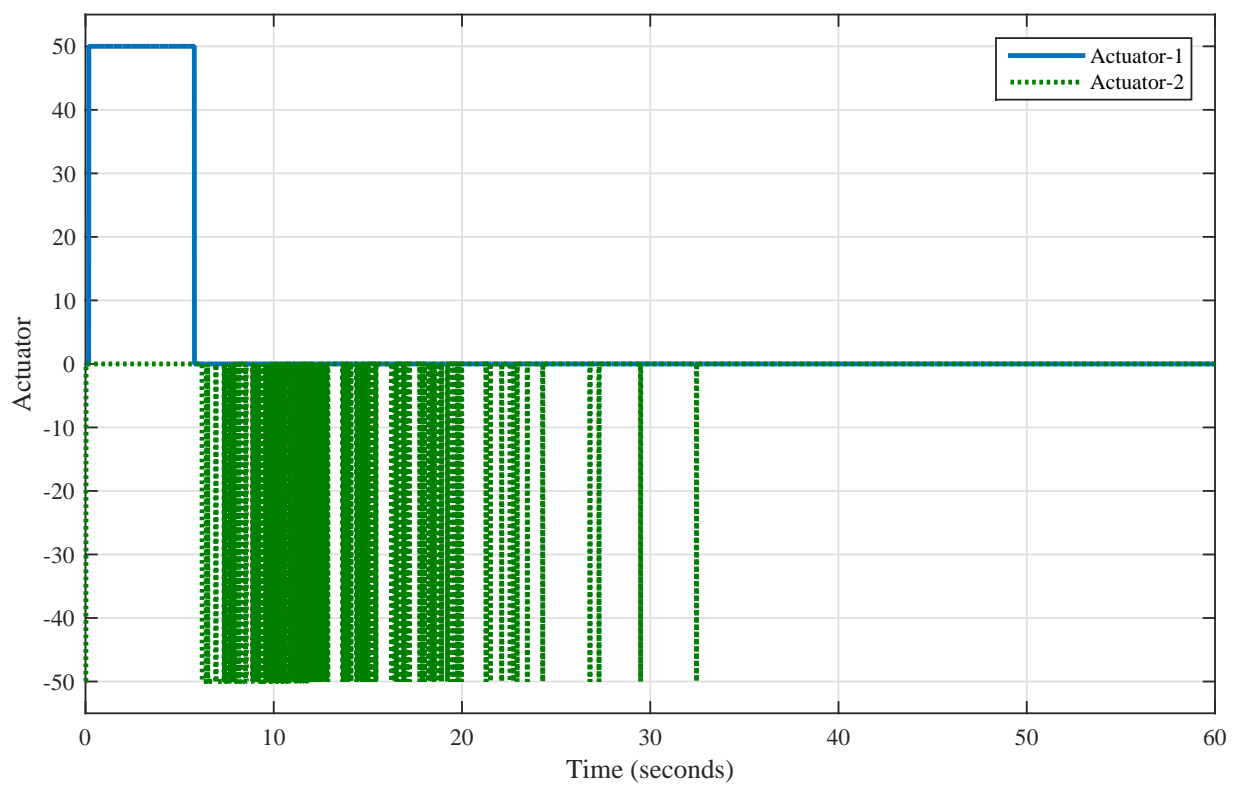

FIGURE 6. Position Control: Actuator response.

prediction horizon $T_{h}=3$ and sampling rate $T_{s}=0.01$. The simulation results for the current scenario can be seen in Figure-7,8,9,10.

In Figure-7 we can see the position of the passive body and the two satellites respectively. In Figure- 8 we can see the velocities of three bodies required to achieve the required position control. The controller output generated by the MPC can be seen in Figure-9 while the ON-OFF actuator response can be seen in Figure-10. The actuator response in Figure-10 shows heavy chattering, which is due to the fact that the control signals are both positive and negative at the same time. Such a behaviour of the controller was essential for the position control of the passive body at the 


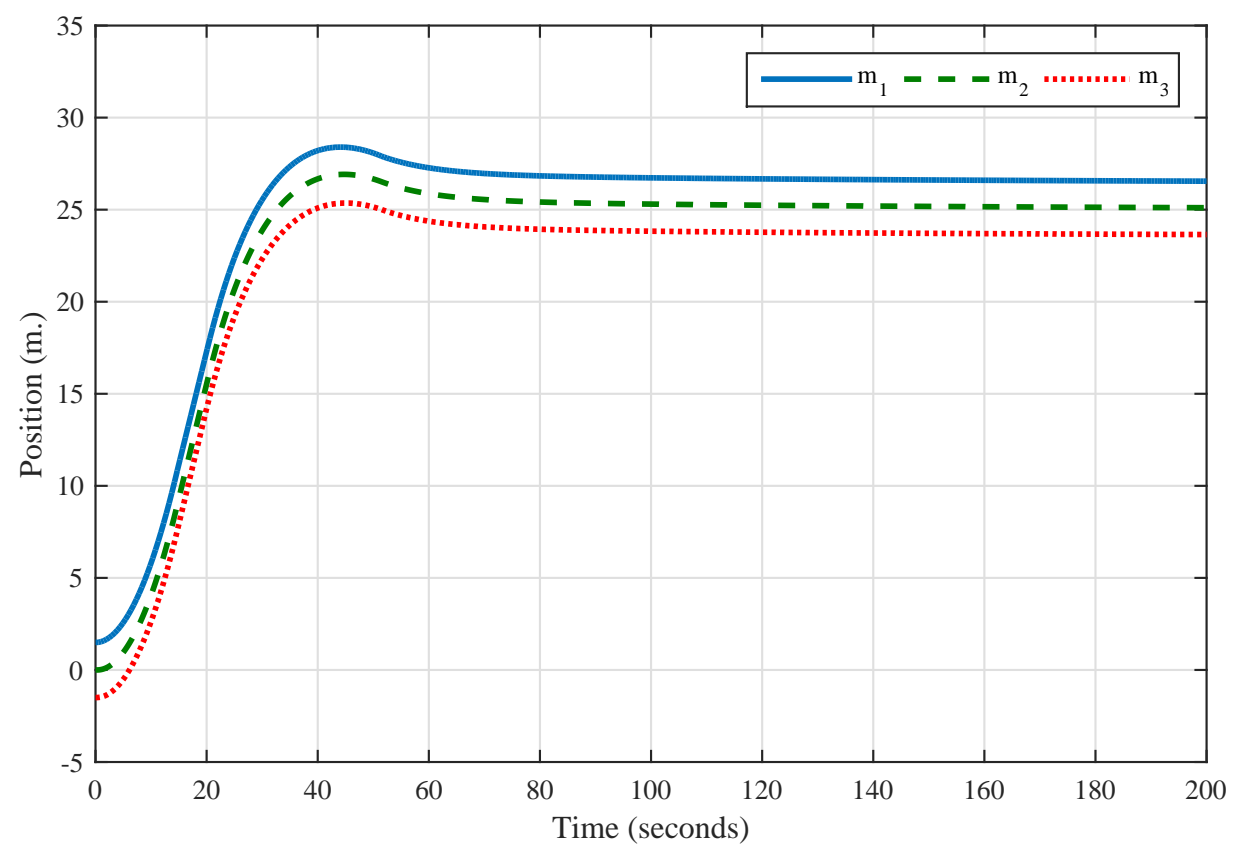

FIGURE 7. Cooperative Position Control: Position response.

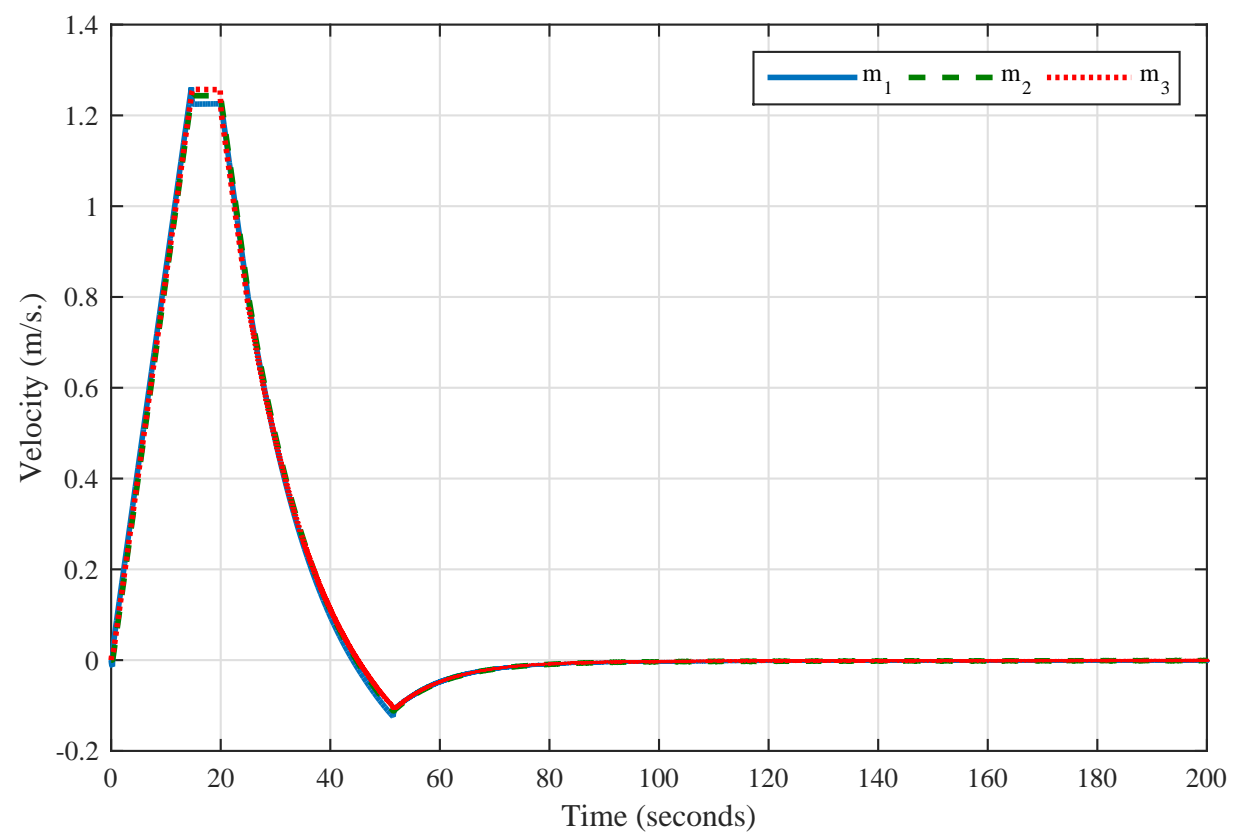

FIGURE 8. Cooperative Position Control: Velocity response.

same time keeping a safe distance to avoid collision. 


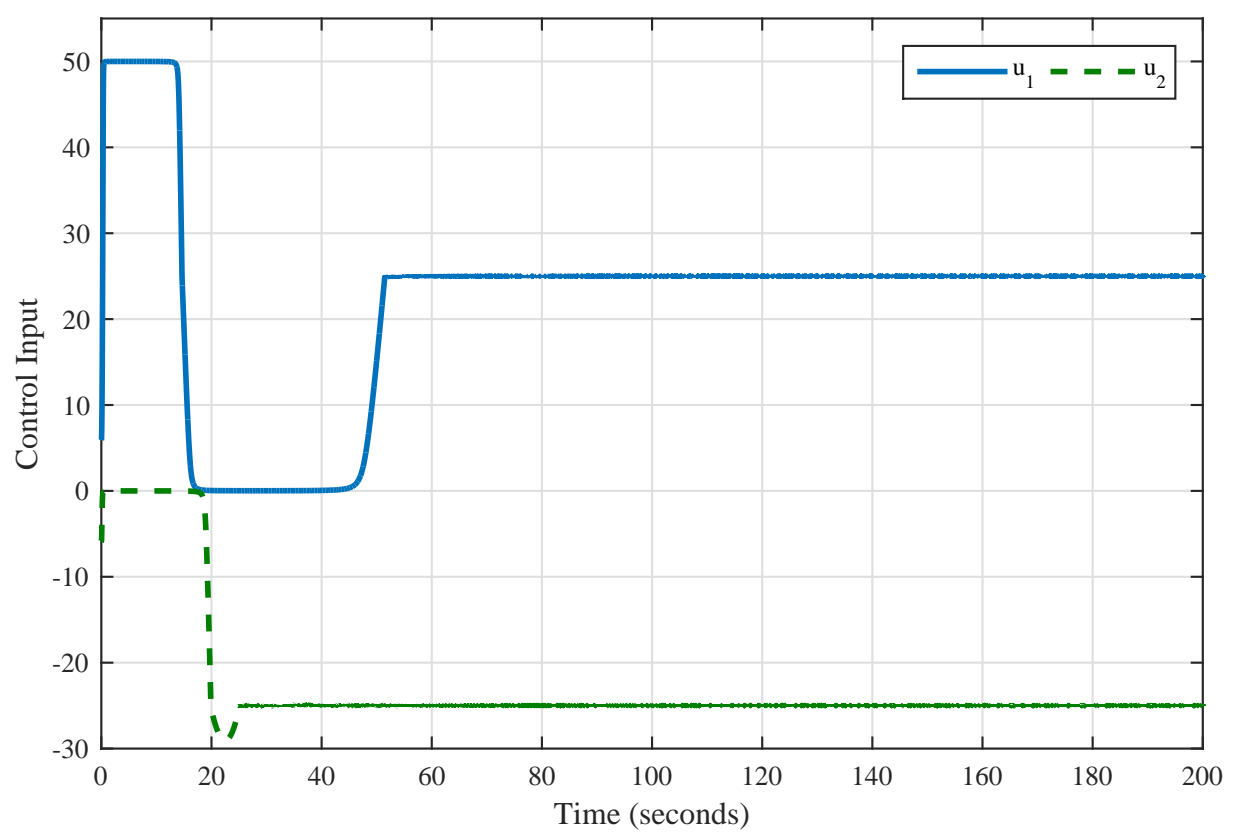

FIGURE 9. Cooperative Position Control: Control Signal.

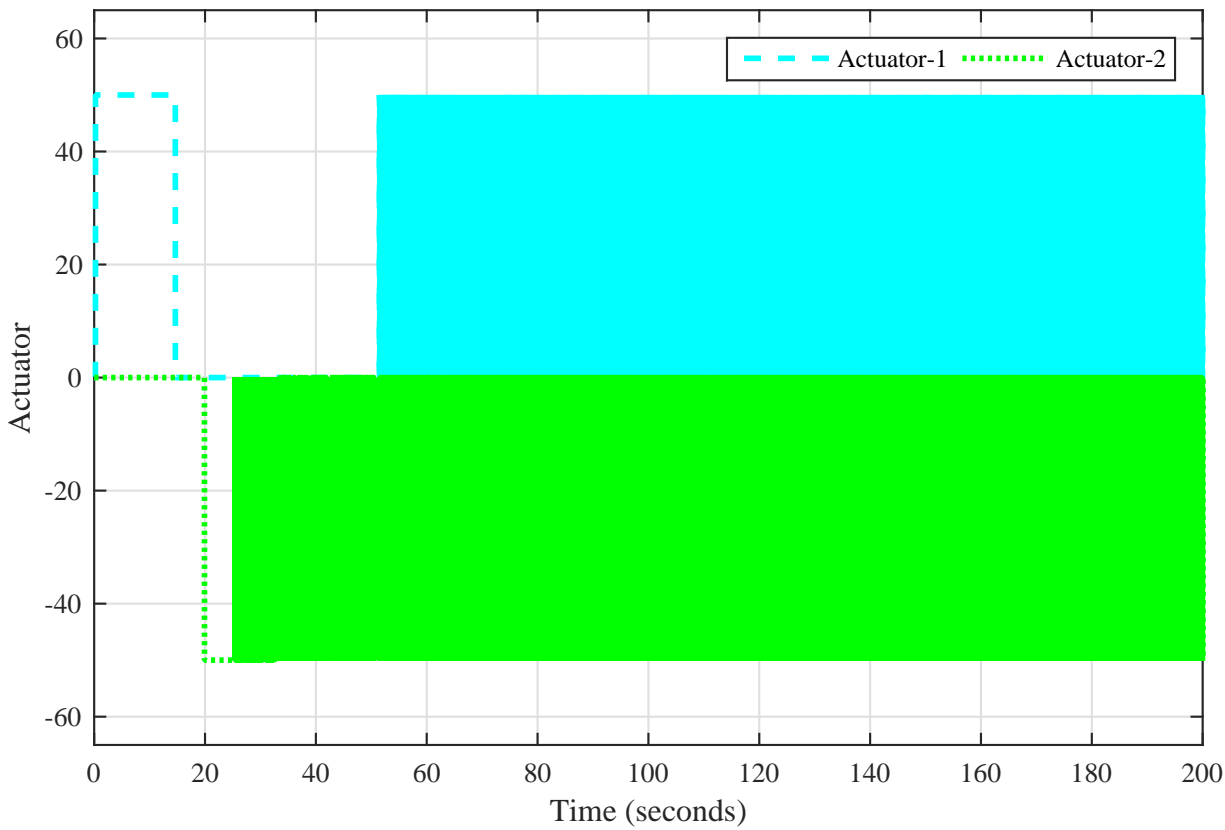

FIGURE 10. Cooperative Position Control: Manipulator Position.

\section{CONCLUSION}

The problem of manipulation of passive objects in space using space robots is discussed. The dynamic models of robotic servicers attached to passive body is also introduced. The Model Predictive Control technique was introduced briefly and successfully applied to two scenarios where a passive body is displaced by two servicers rigidly attached and then the case where the two servicers are attached to the passive body through a manipulator. In both the cases 
the Model Predictive Control Technique proves suitable. The future perspective of the current solution includes the development of MPC based solution to consider position control with energy efficiency and avoiding chattering in the ON-OFF actuators.

\section{REFERENCES}

[1] G. Rekleitis and E. Papadopoulos, "Towards passive object on-orbit manipulation by cooperating free-flying robots," in Robotics and Automation (ICRA), 2010 IEEE International Conference on (2010), pp. 2247-2252.

[2] K. Yoshida, "Ets-vii flight experiments for space robot dynamics and control," in Experimental Robotics VII, edited by D. Rus and S. Singh (Springer Berlin Heidelberg, Berlin, Heidelberg, 2001), pp. 209-218.

[3] E. O. Portal, "Orbital express," aug (2016-22-8).

[4] S. Moosavian, R. Rastergarij, and E. Papadopoulos, AIAA Journal of Guidance, Control and Dynamics 28, 939-947 (2008).

[5] J. Everist, K. Mogharei, H. Suri, N. Ranasinghe, B. Khoshnevis, P. Will, and W.-M. Shen, "A system for in-space assembly," in Intelligent Robots and Systems, 2004. (IROS 2004). Proceedings. 2004 IEEE/RSJ International Conference on, Vol. 3 (2004), pp. 2356-2361 vol.3.

[6] C. Toglia, F. Kennedy, and S. Dubowsky, Autonomous Robots 31, 209-221 (2011).

[7] G. Rekleitis and E. Papadopoulos, "On on-orbit passive object handling by cooperating space robotic servicers," in 2011 IEEE/RSJ International Conference on Intelligent Robots and Systems (2011), pp. 595-600.

[8] G. Rekleitis and E. Papadopoulos, IEEE Transactions on Aerospace and Electronic Systems 51, 802814April (2015).

[9] T. Ohtsuka, Automatica 40, 563 - 574 (2004).

[10] S. A. Sajadi-Alamdari, H. Voos, and M. Darouach, "Nonlinear model predictive extended eco-cruise control for battery electric vehicles," in 2016 24th Mediterranean Conference on Control and Automation (MED) (2016), pp. 467-472.

[11] J. Dentler, S. Kannan, M. A. O. Mendez, and H. Voos, "A real-time model predictive position control with collision avoidance for commercial low-cost quadrotors," in 2016 IEEE Multi-Conference on Systems and Control (MSC 2016) (2016).

[12] H. Seguchi and T. Ohtsuka, "Nonlinear receding horizon control of an rc hovercraft," in Control Applications, 2002. Proceedings of the 2002 International Conference on, Vol. 2 (2002), pp. 1076-1081 vol.2.

[13] H. Seguchi and T. Ohtsuka, International Journal of Robust and Nonlinear Control 13, 381-398 (2003).

[14] J. Dentler, S. Kannan, M. A. O. Mendez, and H. Voos, "A modularization approach for nonlinear model predictive control of distributed fast systems," in 2016 24th Mediterranean Conference on Control and Automation (MED) (2016), pp. 292-297.

[15] T. Ohtsuka, "symlab: cgmres source code," sep (2015-09-4). 